\title{
Excitation spectrum of mesoscopic proximity structures
}

\author{
S. Pilgram, ${ }^{1}$ W. Belzig, ${ }^{2}$ and C. Bruder ${ }^{1}$ \\ ${ }^{1}$ Departement Physik und Astronomie, Universität Basel, Klingelbergstrasse 82, CH-4056 Basel, Switzerland \\ ${ }^{2}$ Theoretical Physics Group, Delft University of Technology, Lorentzweg 1, NL-2628 CJ Delft, The Netherlands
}

(Received 31 May 2000)

\begin{abstract}
We investigate one aspect of the proximity effect, viz., the local density of states of a superconductor-normal metal sandwich. In contrast to earlier work, we allow for the presence of an arbitrary elastic mean free path in the structure. The superconductor induces a gap in the normal-metal spectrum that is proportional to the inverse of the elastic mean free path $l_{\mathrm{N}}$ for rather clean systems. For a mean free path much shorter than the thickness of the normal metal, we find a gap size proportional to $l_{\mathrm{N}}$ that approaches the behavior predicted by the Usadel equation (diffusive limit). We also discuss the influence of interface and surface roughness, the consequences of a nonideal transmittivity of the interface, and the dependence of our results on the choice of the model of impurity scattering.
\end{abstract}

\section{INTRODUCTION}

A normal metal in good metallic contact to a superconductor acquires superconducting properties such as infinite conductance and the Meissner effect (see Ref. 1, and references therein). This so-called proximity effect has been extensively studied in the past decade mainly in the two limiting cases of fully ballistic propagation and diffusive motion. Whereas the first may be realized experimentally in twodimensional electron gases, the latter is realized in the most common samples made of semiconducting or structured metallic films.

Although the ballistic and the diffusive cases provide useful bounds, real-world mesoscopic samples are often in the intermediate regime. For instance, the diamagnetic response of mesoscopic proximity cylinders (superconducting wires covered by a normal metal) has attracted a lot of experimental $^{2-4}$ and theoretical ${ }^{5-8}$ interest. It turned out that the experimental results could only be understood by considering intermediate mean free paths ${ }^{4}$. Previous theoretical analyses in the clean $\operatorname{limit}^{6}$ and the dirty limit ${ }^{5,7}$ were not able to explain the experimental results quantitatively. Only recently was it shown that a qualitative different behavior emerges in the regime of intermediate mean free path. ${ }^{8}$ With the help of this calculation a quantitative agreement with the experimental results could be obtained ${ }^{4}$ and it was possible to determine the mean free path $l_{\mathrm{N}}$.

The precise determination of the degree of disorder is relevant for a characterization of the samples. It may serve as a basis for the understanding of the experimental observation of a low-temperature paramagnetic reentrance effect. ${ }^{2,3}$ This has already stimulated theoretical suggestions that orbital currents might lead to a paramagnetic contribution. ${ }^{9,10}$ These currents depend on the degree of disorder in the normal metal and further investigations are necessary to quantify the influence of disorder.

In the present work, we want to focus on another aspect of the proximity effect, viz., on the change of the excitation spectrum of a normal metal connected to a superconductor. In particular, we are interested in the changes of the spectrum due to disorder.
In this paper, we report on a comprehensive study on the density of states in a moderately dirty proximity sample. The paper is organized as follows. In the next section we introduce the geometry and the parameters of our model. In Sec. III we present results for the density of states for intermediate mean free paths and different models of disorder. Finally, we discuss the effect of rough surfaces and rough interfaces in Sec. IV.

\section{GEOMETRY AND MODEL}

The sample geometry that we have in mind is shown in Fig. 1: we consider a slab geometry in which a normal-metal layer of thickness $d$ is connected by an ideal interface to a superconductor. The outer surface of the sandwich is supposed to be specularly reflecting. In the following, we will discuss the local density of states (LDOS) $N(E, x)$ of this structure for a variety of physical situations. The quasiclassical formulation of superconductivity is most suitable to calculate the LDOS. To this end we have solved the real-time version of the Eilenberger equation ${ }^{11,12}$

$$
-\mathbf{v}_{\mathbf{F}} \nabla \hat{g}\left(\mathbf{v}_{\mathbf{F}}, \mathbf{r}, E\right)=\left[-i E \hat{\tau}_{3}+\Delta(\mathbf{r}) \hat{\tau}_{1}+\hat{\sigma}(\mathbf{r}, E), \hat{g}\left(\mathbf{v}_{\mathbf{F}}, \mathbf{r}, E\right)\right]
$$

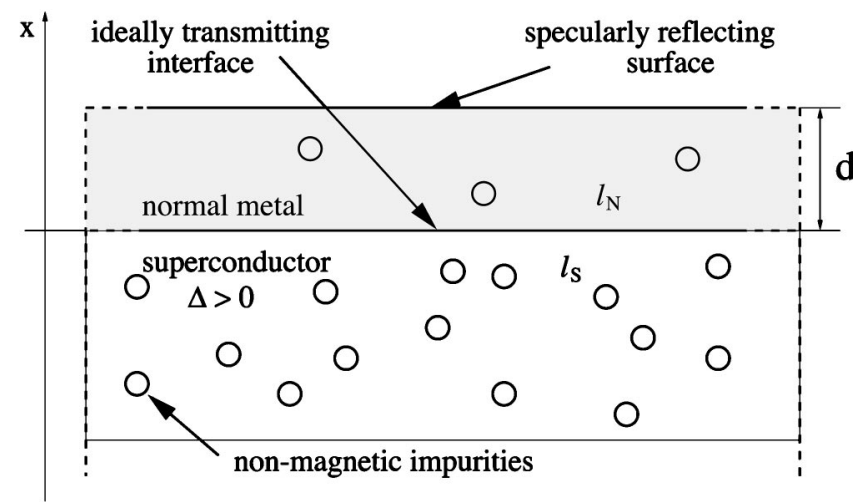

FIG. 1. Geometry of the SN interface. $l_{N}\left(l_{S}\right)$ is the elastic mean free path of the normal (superconducting) part. In the first part of the paper, the interface is supposed to be ideal and the surface specularly reflecting. 
for the quasiclassical $2 \times 2$ matrix Green's function $\hat{g}$ numerically (see Ref. 1 and Appendix A for additional details of this method). Here and in the following, $\hbar$ is set to one except for the final results. In Eq. (1), $\Delta$ is the pair potential and $\hat{\sigma}$ the impurity self-energy which has to be determined self-consistently by a scattering matrix equation. The Pauli matrices $\hat{\tau}_{i}$ are used as a basis for the $2 \times 2$ matrix equation, $\mathbf{v}_{\mathbf{F}}$ is an arbitrarily oriented unit vector times the Fermi velocity. The quasiclassical Green's function is normalized according to

$$
\hat{g}^{2}\left(\mathbf{v}_{\mathbf{F}}, \mathbf{r}, E\right)=1 .
$$

In principle, the pair potential $\Delta$ has to be determined selfconsistently in the superconductor, whereas it vanishes by definition in the normal metal. Since we are mainly interested in the properties of the normal metal in the limit $d$ $\gg \xi_{0}$, where $\xi_{0} \equiv \hbar v_{\mathrm{F}} / \Delta$ is the coherence length of the superconductor, the spatial dependence of the pair potential in the superconductor plays no role. Thus, we approximate the pair potential by a step function $\Delta(\mathbf{r})=\Delta \theta(-x)$.

The effect of impurities give rise to the self-energy $\hat{\sigma}(\mathbf{r})$. In general the impurity self-energy can be found from a solution of a $t$-matrix equation. ${ }^{13}$ In our particular case this equation can be solved and presented as

$$
\hat{\sigma}(\mathbf{r}, E)=\frac{n_{\text {imp }}(\mathbf{r}) v^{2}(\mathbf{r}) N_{0}\langle\hat{g}(\mathbf{r}, E)\rangle}{1+v^{2}(\mathbf{r}) N_{0}^{2}\langle\hat{g}(\mathbf{r}, E)\rangle^{2}} .
$$

This impurity self-energy contains two parameters, the impurity concentration $n_{\text {imp }}$ and the strength of the scattering potential $v(\mathbf{r})$ (that may be spatially dependent if there are different impurities in different parts of the sample). Additionally, the normal-metal density of states at the Fermi level $N_{0}$ enters into Eq. (3). Angular brackets denote averaging over the Fermi surface.

In the rest of the paper we will mostly use the Born approximation valid in the limit of weak scattering, where the denominator in Eq. (3) can be neglected:

$$
\hat{\sigma}(\mathbf{r}, E)=\frac{1}{2 \tau_{\text {imp }}(\mathbf{r})}\langle\hat{g}(\mathbf{r}, E)\rangle .
$$

Here, we have introduced the elastic scattering time $\tau_{\text {imp }}$ $=\left[2 n_{\text {imp }}(\mathbf{r}) v^{2}(\mathbf{r}) N_{0}\right]^{-1}$ which is related to the mean free path $l=v_{\mathrm{F}} \tau_{\text {imp }}$. Since we will also consider inhomogeneous impurity distributions (on a mean level), we still allow for a spatial dependence of elastic scattering time and mean free path.

The set of equations (1)-(4) has been solved numerically. The integration of Eq. (1) was performed using the Riccati parametrization $^{14}$ (see Appendix A1). The self-energies had to be determined self-consistently for all energies in an iterative scheme. Finally, the local density of states is given by

$$
N(E, \mathbf{r})=N_{0} \operatorname{Re}\left\langle\frac{1}{2} \operatorname{Tr} \hat{\tau}_{3} \hat{g}(\mathbf{r}, E)\right\rangle .
$$

Before we discuss our results, let us briefly summarize the known spectral properties in the limiting cases of clean and dirty limit. The case of a clean normal metal was already

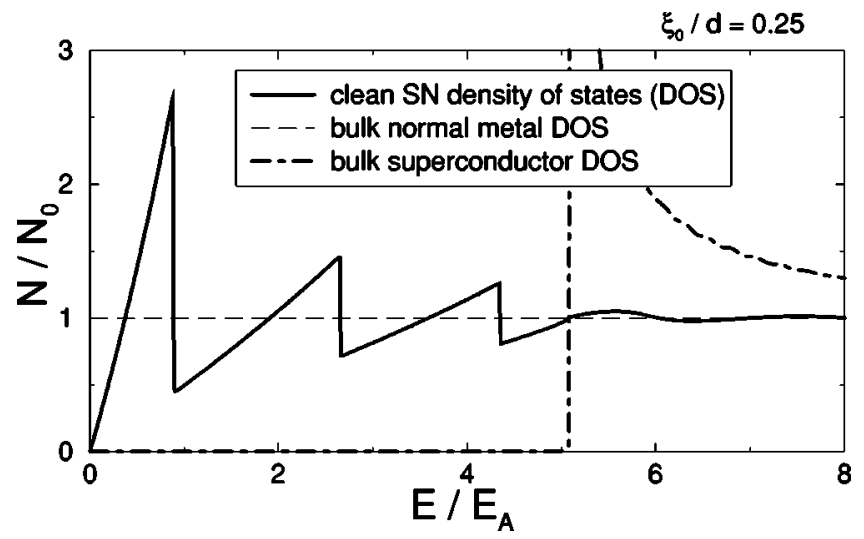

FIG. 2. Density of states of a clean normal metal connected to a superconductor. In the clean case, $N(E, x)=N(E)$, i.e., there is no dependence on the spatial coordinate. The coupling to the superconductor leads to a linear suppression of the DOS at the Fermi energy. Here, we chose $\Delta=5 E_{A}$.

discussed in the 1960's: the LDOS is independent of the location in the normal metal, vanishes at the Fermi energy and rises linearly close to it. ${ }^{15,16}$ Its peculiar zig-zag from is shown in Fig. 2. The characteristic energy determining the jumps in the spectrum is the Andreev energy $E_{\mathrm{A}}$. This characteristic energy follows from a semiclassical quantization condition for the Andreev bound states. According to this rule we have to add the phases accumulated along a trajectory burying a bound electron-hole state. This phase difference is given by twice the phase shift of an Andreev reflection at the superconductor, which at $E \ll \Delta$ is given by $\pi / 2$. On the path through the normal metal both electron and hole accumulate an additional shift of $2 \times 2 E d / v_{\mathrm{F}}$, proportional to twice the time spent in the normal metal. Adding all contributions to the semiclassical phase and requiring it to be an integer multiple of $2 \pi$ leads to the characteristic energy of the lowest level

$$
E_{\mathrm{A}}=\frac{\hbar \pi v_{\mathrm{F}}}{4 d} .
$$

The subsequent levels are (approximately) equidistant with level spacing $2 E_{\mathrm{A}}$ as can be seen from Fig. 2 (the deviations from the ideal level spacing are caused by the finite value of $\Delta \approx 5 E_{\mathrm{A}}$ ).

Another well-known result on the spectrum has been obtained in the dirty (diffusive) limit. ${ }^{17,18}$ In this case, the LDOS is characterized by a minigap in the spectrum that is of the order of the Thouless energy $E_{\mathrm{Th}}=\hbar D / d^{2}$, here $D$ is the diffusion constant of the normal metal and $d$ its thickness. The LDOS of a mesoscopic superconductor-normalmetal sample was determined experimentally ${ }^{19}$ in the presence of a magnetic field, and our theory ${ }^{18}$ led to a satisfactory understanding of those experimental results.

The induced minigap has also been discussed by fieldtheoretic means, see Ref. 20. The relation of this gap to quantum chaos is discussed in Ref. 21.

A qualitative picture of the formation of the minigap follows from considering the Andreev bound states in the normal layer. An electron and hole traversing a diffusive trajectory in the time reversed direction are transformed into each other by Andreev reflection when hitting the superconductor. 


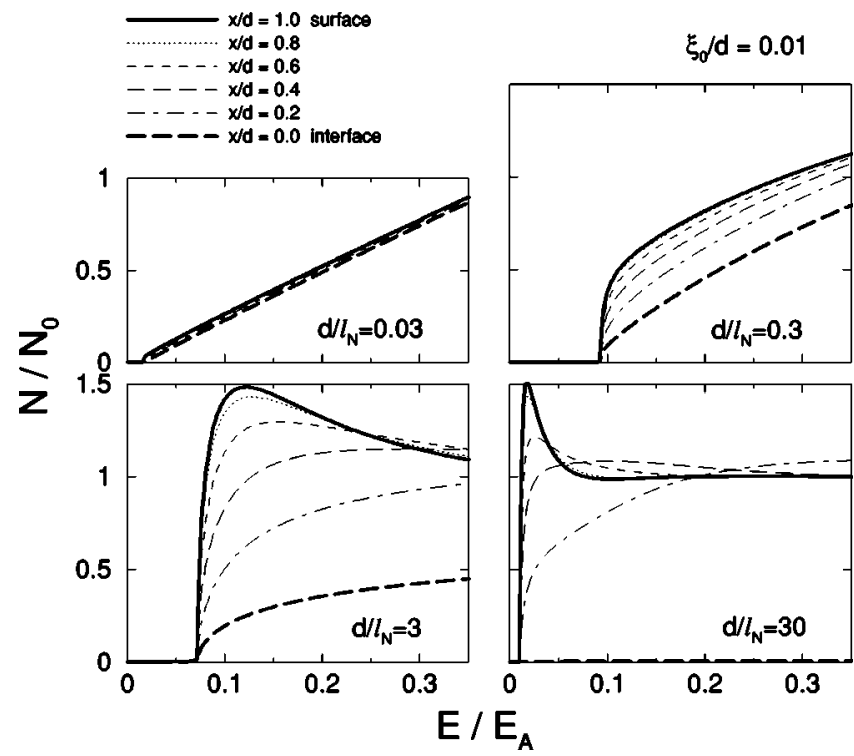

FIG. 3. LDOS $N(E, x)$ for different ratios of mean free path $l_{N}$ to normal-layer thickness $d$. The minigap is constant throughout the normal metal, but the energy dependence of the LDOS changes with location.

The coherent superposition of two subsequent reflections result in a bound state. The phase shift due to Andreev reflection is similar as in the clean limit discussed above. During the motion the two quasiparticle gain an additional phase shift $2 E \delta t$, where $\delta t$ is the time spent in the normal metal region and $2 E$ is the energy difference of electron and hole. In a diffusive system the typical time spent in the normal metal is $\sim 2 d^{2} / D$, i.e., twice the time to diffuse a distance $d$. This time results in a typical lower bound to the energy $\sim \pi D / 4 d^{2}$ above which constructive interference is possible. The minigap is thus expected to be approximately $E_{\mathrm{g}}$ $\approx \pi \hbar D / 4 d^{2}=0.785 \hbar D / d^{2}$. The numerically exact expression for the minigap in the dirty case is given by $E_{\mathrm{g}}$ $=0.780 \hbar \mathrm{D} / \mathrm{d}^{2}$, where the numerical factor is the solution of a transcendental equation (see Appendix B). This result is in very good agreement with the estimate given before corroborating the simple picture of Andreev bound states.

\section{BULK DISORDER IN $N$}

How is the linear rise of the LDOS for the clean system transformed into the minigap in the diffusive system as a function of mean free path? To answer this question, we have solved the real-time Eilenberger equation including an impurity self-energy of the form $\hat{\sigma}=\langle\hat{g}\rangle / 2 \tau_{\text {imp }}$ (Born approximation). The impurity self-energy was determined in a self-consistent way.

We find ${ }^{22,23}$ that a gap forms at arbitrarily small impurity concentrations. This is shown in Fig. 3: even for values of the elastic mean free path $l_{N}$ that are 30 times larger than the normal-layer thickness, the formation of the low-energy gap is clearly visible. The gap increases with $1 / l_{N}$, saturates for $l_{N} \sim d$, and then decreases again as expected from the dirtylimit theory since $D \sim v_{F} l_{N}$, see Fig. 4. The gap does not depend on the location in the normal metal as can be seen in Fig. 3, i.e., it is a global feature. The shape of the LDOS, i.e.,

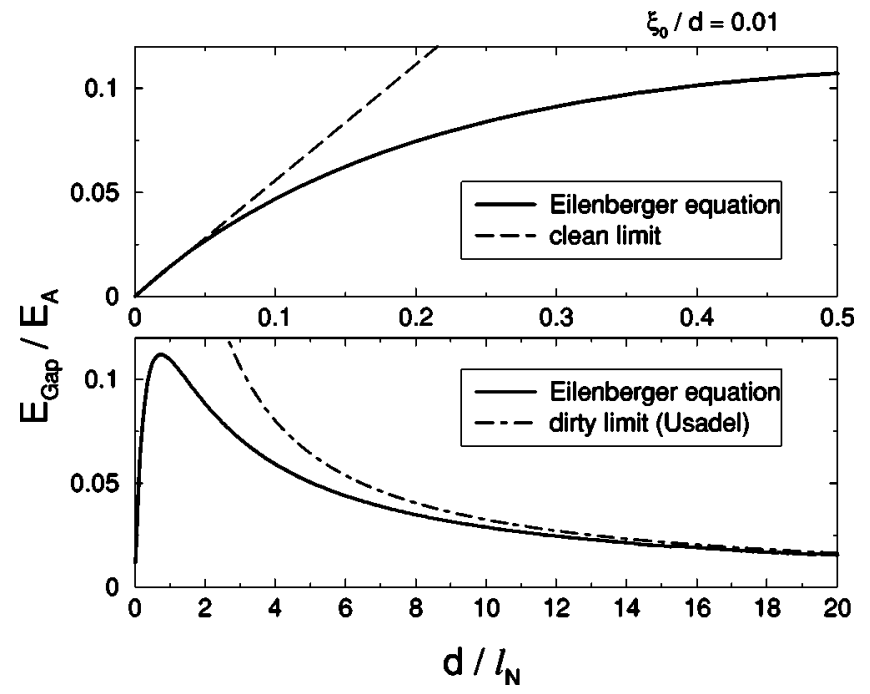

FIG. 4. Size of the minigap obtained by numerically solving the Eilenberger equation.

its dependence on energy, however, varies on traversing the normal layer.

The existence of a minigap is in line with qualitative considerations given by McMillan. ${ }^{16} \mathrm{He}$ argued that-quite generally - the density of states should show a gap of order of the inverse of the escape time, i.e., the time an electron spends in the normal layer before being Andreev-reflected. If we replace the escape time by the scattering time in the (almost) clean system and by the diffusive escape time in the dirty system, we obtain the nonmonotonous behavior shown in Fig. 4.

We have also replaced the Born model for impurity scattering by the unitary limit which follows from the $t$-matrix approximation for $s$-wave scattering Eq. (3) in the limit $v N_{0} \gg 1$. To facilitate a comparison we used for the parameter $n_{i} / N_{0}$, characterizing the strength of the impurity selfenergy in the unitary limit, the value of $1 / 2 \tau_{\text {imp }}$ from the Born approximation. Figure 5 shows the minigap for both the Born and the unitary approximation. The minigap is slightly reduced in the unitary limit, but its functional dependence on the mean free path is practically unchanged.

\section{INTERFACE AND SURFACE ROUGHNESS}

Real-life interfaces and surfaces of typical proximity samples are rough (for an example, see the photograph in the

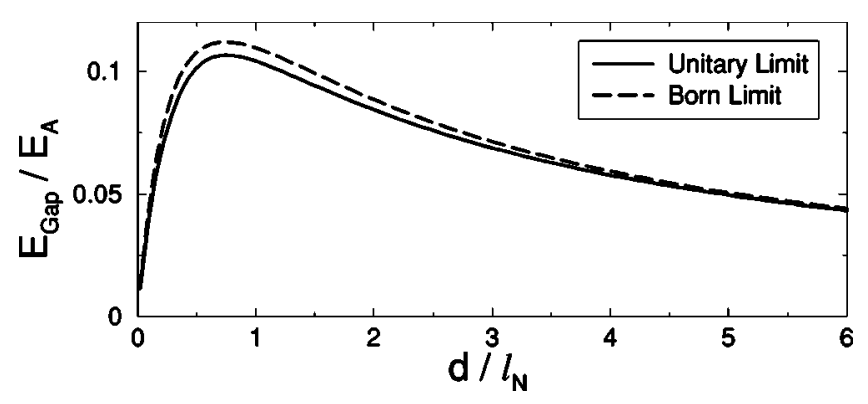

FIG. 5. Dependence of the induced minigap on bulk disorder for two models of impurity scattering, viz., Born and $t$-matrix approximation. The differences are insignificant, i.e., the minigap is stable and is not dependent on the choice of the Born approximation. 


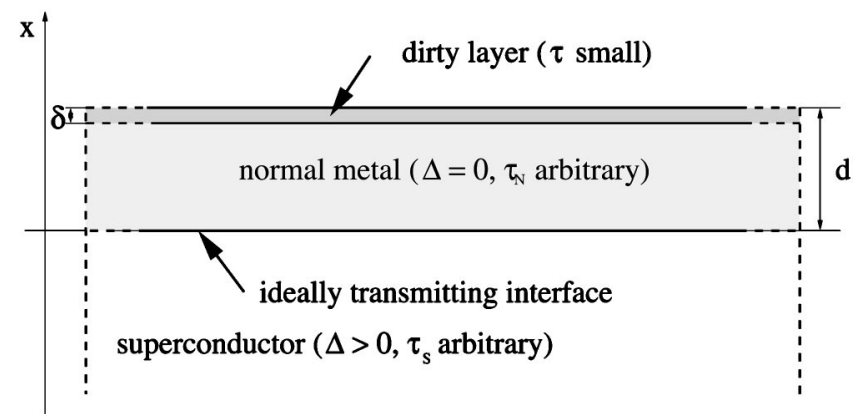

FIG. 6. We model a rough surface (or interface) by replacing it by a thin dirty layer. The scattering time $\tau$ in this layer and its thickness are chosen such as to lead to the proper scattering characteristics of a rough surface.

second paper of Ref. 2). In the quasiclassical language such a roughness will lead to a mixture of different trajectories, thus smearing out the singular Andreev bound states on a given path. A convenient way to include this effect in the quasiclassical formalism is to model the rough surface by a thin dirty layer which covers the inner side of the surface, see Fig. 6. The thickness $\delta$ of the layer should be taken to be much smaller than all characteristic lengths of the rest of the system (in our case these are the thickness of the normal metal $d$ and its mean free path $l_{N}$ ). The disorder in the layer is included by an impurity self-energy with a mean free path $l_{\text {layer }}$. At the outer surface inside the dirty layer specular reflection is assumed and the Green's functions in the dirty layer are continuously connected to the ones in the normal metal. Under these conditions the ratio $l_{\text {layer }} / \delta$ is the only parameter measuring the roughness of the real-life interface.

Similarly the interface roughness between the normal metal and the superconductor is modeled by a thin dirty layer residing now at the interface. The layer is characterized by the same parameters as before. Now we have continuity of the Green's function on both sides of the dirty layer.

It is clear that this model for roughness should be taken with care, since it is by no means microscopically justified. The parameters characterizing the dirty layer are not related to the real parameters of the interface. It is, however, clear that this model covers the essential properties of a realistic rough surface, i.e., it couples classical trajectories which would be uncoupled for a specular interface. Thus it leads to an averaging over spectral quantities from different regions of the system in the same way as a realistic system does on average. One should, however, keep in mind, that we can only determine averages over many characteristic lengths on the roughness in this way. For example, in small cavities with a characteristic length of roughness which is comparable to the size of the system, such an averaging is not appropriate and fluctuations may become important.

\section{A. Surface disorder}

The result of a calculation for a rough outer surface is shown in the left plot of Fig. 7. We have kept the roughness of the surface fixed in this plot and varied the bulk disorder. Only the LDOS at the outer surface is plotted. In our example, the roughness parameter is such that there are on average two scattering events in the dirty layer, i.e., the outer
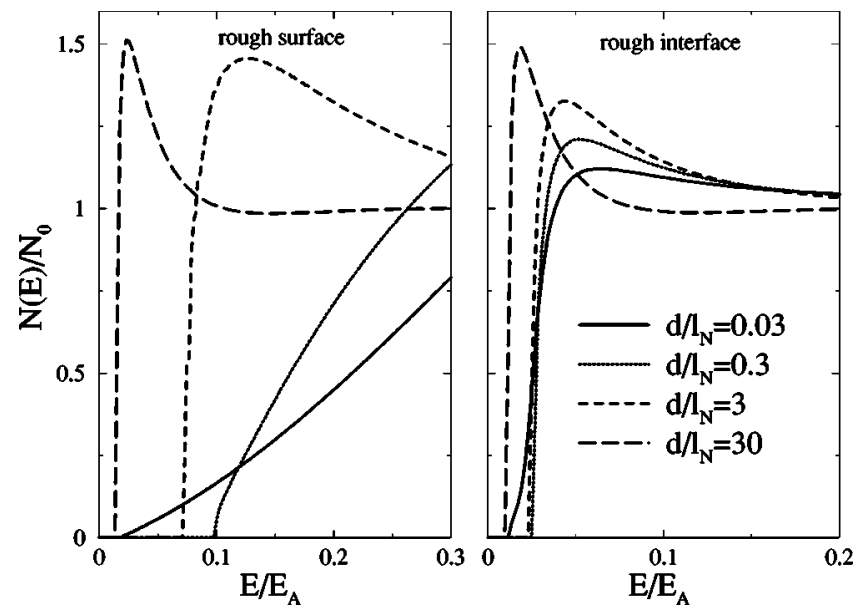

FIG. 7. LDOS at the outer surface $(x=d)$ under the influence of surface disorder (left plot) or interface disorder between superconductor and normal metal (right plot). The thickness of the layer is chosen to be $\delta=10^{-5} d$ and the mean free path in the layer is $l_{\text {layer }}=\delta / 2$. The different curves in each plot are for different degrees of disorder in the normal metal as indicated in the right plot. A comparison with Fig. 3 shows that the size of the minigap is practically conserved in the left plot, whereas the non-ideal interface leads to a decreased minigap in the right plot.

surface is definitely nonspecular. The induced minigap is not very susceptible to the presence of the surface roughness as follows from a comparison with Fig. 3. Using McMillan's argument that the minigap should be inversely proportional to the escape time, we can qualitatively understand this behavior. The effect of the scattering by the rough surface leads to a reduction of the number of the shorter trajectories, but the length of the shortest trajectory itself is not changed. This can be most clearly seen for an intermediate bulk disorder $l \approx 3 d$, where the sharp increase at the minigap for the specular surface is nearly absent. The rough surface also leads to a change in the spatial form of the density of states, which is not shown in the plot.

We remark in passing that surface roughness without bulk disorder will not lead to the formation of a minigap, since there will be no upper cutoff for the trajectory lengths in this case. Nevertheless it influences the energy dependence of the LDOS, by virtue of a similar effect as mentioned previously. Due to the reduction of the number of the longer trajectories spectral weight is shifted to higher energies. This can already be seen from the solid curve in the left plot Fig. 7, where the linear energy dependence in the case of specular reflection has turned into some weaker energy dependence.

\section{B. Interface disorder}

In many experimental situations the interface between a normal metal and a superconductor will be nonideal in the sense that either transport through the interface is not along classical trajectories or the electrons are partially reflected. This can be due to either an oxide layer or an alloy in the interface region, and/or due to differences in effective masses or Fermi velocities between the superconductor and the normal metal. The generic properties of these disordered interfaces can be included in the quasiclassical formalism by a thin dirty layer located at the interface. This model will 


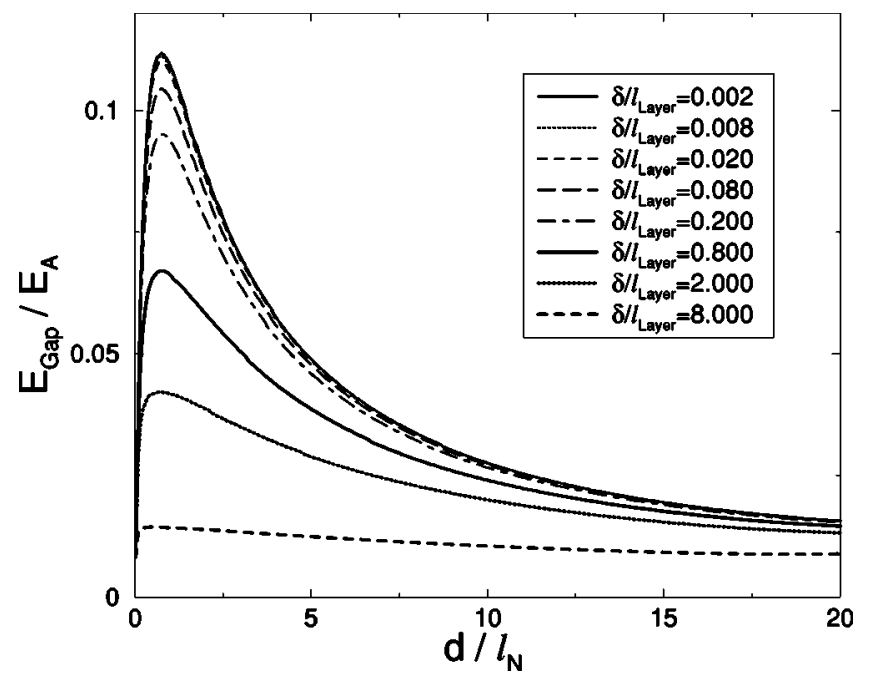

FIG. 8. Influence of interface roughness (parametrized by $\left.\delta / l_{\text {layer }}\right)$ on the induced minigap in the normal layer. In contrast to surface roughness, interface roughness leads to a pronounced suppression of the minigap. In particular roughness parameters larger than 1 lead to a strong suppression of the minigap.

also include the effect of a finite reflection at the interface. Since it is a well known universal property of disordered conductors to have a bimodal distribution of transmission eigenvalues (see, e.g., Ref. 24), we believe that the model of such an disordered layer will cover most of the characteristics of rough contacts.

In the right plot of Fig. 7 we show the effect of a thin dirty layer at the interface between superconductor and normal metal. The interface is chosen to have a mean free path $l_{\text {layer }}=\delta / 2$, similar to the previous case of the rough surface. This is supposed to mimic a rough interface between the metals with equal Fermi velocities. We find a significant reduction of the minigap. This is clearly seen from comparison with the left plot of Fig. 7 and shown as a function of the interface roughness parameter in Fig. 8. For roughness parameters $\delta / l_{\text {layer }}$ smaller than $\approx 1$ the reduction of the minigap is weak. If this parameter is of the order of or larger than 1 the minigap is strongly decreased.

This behavior can be understood by looking at the reflection properties of the rough interface. If the roughness parameter is smaller than 1 the reflectivity will be roughly given by $R \approx \delta / l_{\text {layer }}$, i.e., the probability for a particle to be reflected is given by the ratio of the mean time spent in the layer to the scattering time. As a rough estimate of the effect of the finite reflectivity $R>0$ for the minigap, we use the fact that the mean time that an electron spends in the normal metal will be increased by a factor $1+R+R^{2}+\cdots=1 / T$, where $T$ is the transmission eigenvalue of the interface. Hence, the minigap will be reduced by a factor $T \approx(1$ $\left.-\delta / l_{\text {layer }}\right)$. The influence of finite reflection coefficients in the clean system was also discussed in Ref. 25.

If the interface is strongly disordered, i.e., the roughness parameter is larger than 1 , it behaves more similar to a disordered metal, having a bimodal distribution of transmission eigenvalues. Most of the transmission eigenvalues are close to zero and a few are close to unity allowing for Andreev reflection. The ratio of the number of closed to the number of open trajectories is roughly given by $\delta / l_{\text {layer }}$. Thus, an elec- tron typically is normally reflected by the dirty layer many times until it has the chance to be Andreev reflected. This strongly enhances the length of the trajectories and therefore reduces the minigap roughly by a factor $l_{\text {layer }} / \delta$. This explains the strong suppression of the minigap that sets in for values of the roughness parameter larger than 1 as shown in Fig. 8.

Similar arguments may be used to discuss the effect of impurities in the superconductor, which was so far assumed to be in the clean limit $\left(l_{S} \gg \xi_{0}\right)$. A reduction of the minigap appears in the same manner as described above, if the superconductor is dirty, i.e., has an elastic mean free path $l_{S}$ shorter than the coherence length $\xi_{0}=v_{F} / \Delta_{S}$. The disorder close to the interface acts similarly as the dirty layer at the interface. The role of the "roughness" parameter is now taken over by $\xi_{0} / l_{S}$, since Andreev reflection occurs in this layer of the superconductor. Again the effective length of the trajectory in the normal layer is enhanced by normal reflection at the impurities in the superconductor. Thus, we expect a qualitative similar behavior that the minigap will be reduced by an increase of the disorder in the superconductor.

\section{CONCLUDING REMARKS}

In conclusion, we have studied the local density of states of a proximity sandwich for a variety of situations. At arbitrarily small impurity concentration, a gap opens at the Fermi energy; it is maximal if the elastic mean free path is of the order of the normal-layer thickness. We have numerically calculated this gap and its dependence on surface and interface roughness. Whereas the gap is relatively stable to surface roughness, it is strongly suppressed by interface roughness. We have also investigated the effect of a nonideally transmitting interface. Lastly, we have investigated the influence of different models of impurity scattering (Born vs unitary limit) and shown that the two models lead to a qualitatively similar behavior.

\section{ACKNOWLEDGMENTS}

W.B. would like to thank the "Stichting voor Fundamenteel Onderzoek der Materie", (FOM) and the "Alexander von Humboldt-Stiftung' for financial support.

\section{APPENDIX A: NUMERICAL CALCULATIONS}

For our calculations we use the Riccati parametrization of the Eilenberger equations (see Ref. 14). We represent the Green's function on a trajectory in the form

$$
\hat{g}=\frac{1}{1+a a^{\dagger}}\left(\begin{array}{ll}
1-a a^{\dagger} & 2 a \\
2 a^{\dagger} & a a^{\dagger}-1
\end{array}\right) \text {. }
$$

We have introduced the Andreev amplitudes $a$ and $a^{\dagger}$, which depend on the variables as $\hat{g}$. From the Eilenberger equation (1) one derives two decoupled numerically stable equations of the Riccati type

$$
\begin{gathered}
-\mathbf{v}_{\mathbf{F}} \nabla a=2 \tilde{\omega} a+\tilde{\Delta} * a^{2}-\tilde{\Delta}, \\
\mathbf{v}_{\mathbf{F}} \nabla a^{\dagger}=2 \tilde{\omega} a^{\dagger}+\tilde{\Delta} a^{\dagger 2}-\tilde{\Delta} * .
\end{gathered}
$$


Here, the impurity self-energy is included in $\tilde{\omega}$ and $\tilde{\Delta}$ :

$$
\begin{aligned}
& \tilde{\omega}=-i E+\sigma_{11}(\mathbf{r}), \\
& \tilde{\Delta}=\Delta(\mathbf{r})+\sigma_{12}(\mathbf{r}) .
\end{aligned}
$$

We note that the first equation is stable in an integration in positive direction along the trajectory, whereas the second equation is stable for integration in the opposite direction. In the stable direction the differential equation (A2) is conveniently integrated by a discretization which leads to the very accurate expression

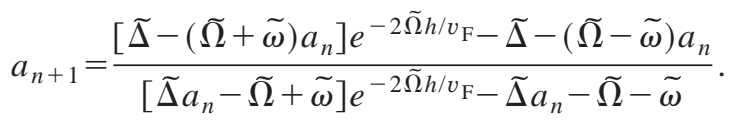

Here, $\widetilde{\Omega}=\left(\widetilde{\Delta}^{2}+\widetilde{\omega}^{2}\right)^{1 / 2}$, and $h$ is the step size.

\section{APPENDIX B: MINIGAP IN THE DIRTY LIMIT}

In this case, it is sufficient to use the Usadel equation ${ }^{26}$

$$
\frac{D}{2} \frac{d^{2}}{d x^{2}} \Theta=\omega \sin \Theta-\Delta \cos \Theta
$$

the dirty-limit expansion of the Eilenberger equation. ${ }^{11,12}$ It contains only one energy scale, the Thouless energy $E_{\mathrm{Th}}$ $=\hbar D / d^{2}$ (apart from the pair potential $\Delta$ ). The minigap that emerges on this scale can be explained by the following argument. The LDOS is given by the real part of $g=\cos \Theta$. If it vanishes, we may write $\cos \Theta=-i \sinh \vartheta$ where $\vartheta$ is real, and the Usadel equation in the normal metal reduces to

$$
\frac{d^{2} \vartheta}{d \xi^{2}}=\frac{2 E d^{2}}{D} \cosh \vartheta
$$

(here, $\xi=x / d$ ). This differential equation is of elliptic type and can be integrated twice. Next, we apply the boundary conditions $\vartheta(\xi=0)=0$ and $(d / d \xi) \vartheta(\xi=1)=0$. The latter accounts for the symmetry of the reflecting surface. The first condition approximates $\vartheta$ by its value in the bulk superconductor at low energies which is justified, if $\Delta$ is sufficiently large. We find that Eq. (B2) is only solvable, if

$$
E<0.780 \hbar D / d^{2}
$$

The minigap of the dirty limit is set by this restriction.
${ }^{1}$ W. Belzig, F.K. Wilhelm, C. Bruder, G. Schön, and A.D. Zaikin, Superlattices Microstruct. 25, 1251 (1999).

${ }^{2}$ P. Visani, A.C. Mota, and A. Pollini, Phys. Rev. Lett. 65, 1514 (1990); A.C. Mota, P. Visani, A. Pollini, and K. Aupke, Physica B 197, 95 (1994).

${ }^{3}$ F.B. Müller-Allinger and A.C. Mota, Phys. Rev. Lett. 84, 3161 (2000).

${ }^{4}$ F. Bernd Müller-Allinger, A.C. Mota, and W. Belzig, Phys. Rev. B 59, 8887 (1999).

${ }^{5}$ P.G. De Gennes, Rev. Mod. Phys. 36, 225 (1964).

${ }^{6}$ A.D. Zaikin, Solid State Commun. 41, 533 (1982).

${ }^{7}$ Y. Oda and H. Nagano, Solid State Commun. 35, 631 (1980).

${ }^{8}$ W. Belzig, C. Bruder, and A.L. Fauchère, Phys. Rev. B 58, 14531 (1998).

${ }^{9}$ C. Bruder and Y. Imry, Phys. Rev. Lett. 80, 5782 (1998).

${ }^{10}$ A.L. Fauchère, W. Belzig, and G. Blatter, Phys. Rev. Lett. 82, 3336 (1999).

${ }^{11}$ G. Eilenberger, Z. Phys. 214, 195 (1968).

${ }^{12}$ A.I. Larkin and Yu.N. Ovchinnikov, Zh. Éksp. Teor. Fiz. 552262 (1968) [Sov. Phys. JETP 26, 1200 (1968)].

${ }^{13}$ L.J. Bucholtz and G. Zwicknagl, Phys. Rev. B 23, 5788 (1981).
${ }^{14}$ N. Schopohl and K. Maki, Phys. Rev. B 52, 490 (1995).

${ }^{15}$ D. Saint-James, J. Phys. (France) 25, 899 (1964).

${ }^{16}$ W.L. McMillan, Phys. Rev. 175, 537 (1968).

${ }^{17}$ A.A. Golubov and M.Yu. Kupriyanov, J. Low Temp. Phys. 70, 83 (1988).

${ }^{18}$ W. Belzig, C. Bruder, and G. Schön, Phys. Rev. B 54, 9443 (1996).

${ }^{19}$ S. Guéron, H. Pothier, N.O. Birge, D. Esteve, and M. H. Devoret, Phys. Rev. Lett. 77, 3025 (1996).

${ }^{20}$ A. Altland, B.D. Simons, and D. Taras-Semchuk, Adv. Phys. 49, 321 (2000).

${ }^{21}$ K.M. Frahm et al., P.W. Brouwer, J.A. Melsen, and C.W.J. Beenakker, Phys. Rev. Lett. 76, 2981 (1996); A. Altland and M.R. Zirnbauer, ibid. 76, 3420 (1996); A. Lodder and Yu.V. Nazarov, Phys. Rev. B 58, 5783 (1998); H. Schomerus and C.W.J. Beenakker, Phys. Rev. Lett. 82, 2951 (1999).

${ }^{22}$ S. Pilgram, Diploma thesis, Universität Basel, 2000.

${ }^{23}$ S. Pilgram, W. Belzig, and C. Bruder, Physica B 280, 442 (2000).

${ }^{24}$ C.W.J. Beenakker, Rev. Mod. Phys. 69, 731 (1997).

${ }^{25}$ Y. Nagato and K. Nagai, J. Phys. Soc. Jpn. 64, 1714 (1995).

${ }^{26}$ K.D. Usadel, Phys. Rev. Lett. 25, 507 (1970). 